\title{
Optimization of L-ornithine
} production in recombinant Corynebacterium glutamicum S9114 by cg3035 overexpression and manipulating the central metabolic pathway

\author{
Bin Zhang ${ }^{1}$, Miao Yu' ${ }^{1}$, Wen-Ping Wei ${ }^{1}$ and Bang-Ce Ye ${ }^{1,2^{*}}$
}

\begin{abstract}
Background: L-Ornithine is an important amino acid with broad applications in pharmaceutical and food industries. Despite lagging L-ornithine productivity and cost reduction, microbial fermentation is a promising route for sustainable $\mathbf{L}$-ornithine production and thus development of robust microbial strains with high stability and productivity is essential.

Results: Previously, we systematically developed a new strain, $\mathrm{SO} 1$ originate from Corynebacterium glutamicum S9114, for L-ornithine production. In this work, overexpression of cg3035 encoding N-acetylglutamate synthase (NAGS) using a plasmid or by inserting a strong $P_{\text {tac }}$ promoter into the chromosome was found to increase L-ornithine production in the engineered C. glutamicum SO1. The genome-based cg3035 modulated strain was further engineered by attenuating the expression of $p t a$ and cat, inserting a strong $\mathrm{P}_{\text {eftu }}$ promoter in the upstream region of glycolytic enzymes such as pfkA, gap, and pyk, and redirecting carbon flux to the pentose phosphate pathway. The final strain with all the exploratory metabolic engineering manipulations produced $32.3 \mathrm{~g} / \mathrm{L}$ of $\mathrm{L}$-ornithine, a yield of $0.395 \mathrm{~g}$ ornithine per g glucose, which was $35.7 \%$ higher than that produced by the original strain $(23.8 \mathrm{~g} / \mathrm{L})$.
\end{abstract}

Conclusion: These results clearly demonstrated that enhancing the expression of NAGS promoted L-ornithine production and provide a promising alternative systematic blueprint for developing L-ornithine-producing C. glutamicum strains.

Keywords: Corynebacterium glutamicum, L-Ornithine, Metabolic engineering

\section{Background}

Chemicals produced by metabolically engineered strains have gained major importance in industrial biotechnology, which is developing as a stimulating field due to the advantages of providing environmentally friendly products to replace petrochemicals [1]. With the development

\footnotetext{
*Correspondence: bcye@ecust.edu.cn

${ }^{1}$ Laboratory of Biosystems and Microanalysis, State Key Laboratory of Bioreactor Engineering, East China University of Science and Technology, Shanghai 200237, China

Full list of author information is available at the end of the article
}

of gene manipulation tools, there is an increasing number of strains such as Escherichia coli, Corynebacterium glutamicum, Bacillus subtilis and others that have been modified to produce economically valuable products. C. glutamicum is a favored industrial microorganism with promising applications in the industrial production of amino acids such as L-glutamate and L-lysine at a million-ton scale in the past decades [2,3]. Recently, increasing studies have used C. glutamicum for producing several products including diamines $[4,5]$, terpene [6], diols [7], and amino acids such as L-isoleucine, L-arginine, and L-ornithine [3]. L-Ornithine is an 
intermediate metabolite in the urea cycle, a key precursor for the biosynthesis of L-citrulline, L-proline, and polyamines, and a non-essential amino acid that plays a critical role in post-traumatic treatment, liver protection [8] and treatment of liver disease, strengthening the heart, and maintaining the working of the immune system [9].

Due to the importance of L-ornithine in human healthpromoting activities, economical and efficient production of ornithine has received much attention in the past years. The major strategy for producing L-ornithine is focused on the enzymatic action of arginase on arginine and microbial fermentation. Metabolic engineering of microorganisms to produce L-ornithine is an attractive alternative due to the high economic cost pressures of using L-arginine for the enzymatic reaction. Recently, several reports have focused on the development of metabolically engineered strains that rapidly convert high concentrations of simple sugars to L-ornithine. Jensen et al. constructed a metabolically engineered C. glutamicum strain that could produce $0.524 \mathrm{~g}$ L-ornithine per $\mathrm{g}$ glucose in CgXII medium through disruption of argFRG and overexpression of $g d h$ and $\operatorname{argCJBD}[10]$. Hwang and Cho modulated the NADPH supply to L-ornithine biosynthesis by inactivating three putative glucose dehydrogenases, which improved the yield of L-ornithine up to $14 \mathrm{~g} / \mathrm{L}$ [11]. Kim et al. developed a high L-ornithine producing $C$. glutamicum strain by disrupting $\operatorname{argF}$, $\arg R$, and $\operatorname{proB}$, and overexpressing the operon $\operatorname{argCJBD}$ from C. glutamicum ATCC 21831. This strain produced $51.5 \mathrm{~g} / \mathrm{L}$ L-ornithine from glucose in a fed-batch culture in a 6.6-L fermenter [12]. Jiang et al. engineered a $C$. glutamicum ATCC 13032-derived strain that produced $24.1 \mathrm{~g} / \mathrm{L}$ L-ornithine in shake flask cultures by genetic modulation and adaptive evolution [13].

In previous study, we developed an engineered strain of C. glutamicum S9114 by deletion of argF, ncgl1221, argR, and putP, attenuation of odhA, proB, and $n c g l 2228$, and overexpression of $l y s E, g d h$, and $\operatorname{argCJBD}$, which produced up to $25 \mathrm{~g} / \mathrm{L}$ of L-ornithine in a shake flask culture $[14,15]$. In the present study, further manipulation to improve L-ornithine production by the genetically engineered C. glutamicum strain was reported (see in Fig. 1). Overexpression of cg3035 using plasmid-based overexpression and promoter insertion experiments for improving L-ornithine production in C. glutamicum SO1 was explored. In addition, the effects of overexpressing the key enzymes in glycolysis, along with attenuation of acetate biosynthesis were evaluated. Next, redirection of the metabolic flux to pentose phosphate pathway was also modulated.

\section{Methods}

\section{Strains and plasmids}

A high L-ornithine producing strain C. glutamicum SO1 (S9114 with deletion of $\operatorname{argF}, n c g l 1221$, argR, and putP, attenuation of odhA, proB, and $n c g l 2228$, and overexpression of lysE, $g d h$, and $\operatorname{argCJBD}$ ) derived from C. $g l u$ tamicum S9114 was used as the original strain for further strain development. The recombinant strains and the plasmid constructed in this study are listed in Table 1. For standard molecular manipulation, E. coli $\mathrm{DH} 5 \alpha$ was used as the host for gene cloning. Luria-Bertani (LB) medium was used to propagate E. coli and C. glutamicum.

\section{DNA manipulation and strain construction}

To construct the recombinant strains, the genomic DNA of strain C. glutamicum S9114 was isolated using a genomic DNA extraction kit (Tiangen, Beijing, China) and employed for DNA fragment amplification. The PCR product and vectors were obtained using the PCR products Purification kit and the mini-plasmid isolation kit (Tiangen, Beijing, China), respectively. The primers used in this study are listed in Additional file 1: Table S1. For introducing modifications into the chromosome of $C$. glutamicum S9114, the suicide vector pK18mobsacB containing the sucrose lethal gene $s a c B$ from Bacillus subtilis was employed by double crossover recombination, as described previously $[16,17]$.

For cg3035 overexpression in the high L-ornithine producing strain $\mathrm{SO} 1$, a widely used strong $\mathrm{P}_{\text {sod }}$ promoter and the open reading frame of $c g 3035$ was amplified and spliced using PCR. The overlapped fragment was ligated

\footnotetext{
(See figure on next page.)

Fig. 1 L-Ornithine biosynthesis metabolic pathways in C. glutamicum and strategies to improve L-ornithine accumulation. The red $\times$ represented this pathways were inactivated. The blue font and arrows represented that pathways were attenuated. The green font and arrows indicated that pathways were overexpressed. The genes encoding enzymes involved in catalytically relevant reactions. pgi, encodes glucose-6-phosphate isomerase; zwf, encodes glucose-6-phosphate dehydrogenase; pgl, encodes 6-phosphogluconolactonase; $t k t$, encodes transketolase; tal, encodes transaldolase; opcA, encodes the putative glucose-6-phosphate dehydrogenase assembly protein; pfkA, encodes ATP-dependent phosphofructokinase; gap, encodes glyceraldehyde 3-phosphate dehydrogenase; $p y k$, encodes pyruvate kinase; pta, encodes phosphotransacetylase; ackA, encodes acetate kinase; cat, encodes succinyl-CoA:acetate CoA-transferase; $n c g l 2228$, encodes a putative branched amino acid transporter protein; odhA, encodes a subunit of 2-ketoglutarate dehydrogenase; $g d h$, encodes glutamate dehydrogenase; ncg/1221, encodes glutamate transporter; proB, encodes gamma-glutamate kinase; cg3035, encodes N-acetylglutamate synthase; argB, encodes $\mathrm{N}$-acetylglutamate kinase; $\operatorname{argC}$, encodes $\mathrm{N}$-acetyl-gamma-glutamylphosphate reductase; argD, encodes acetylornithine aminotransferase; arg $\mathrm{F}$,

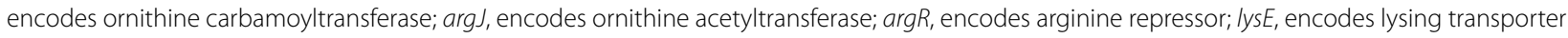




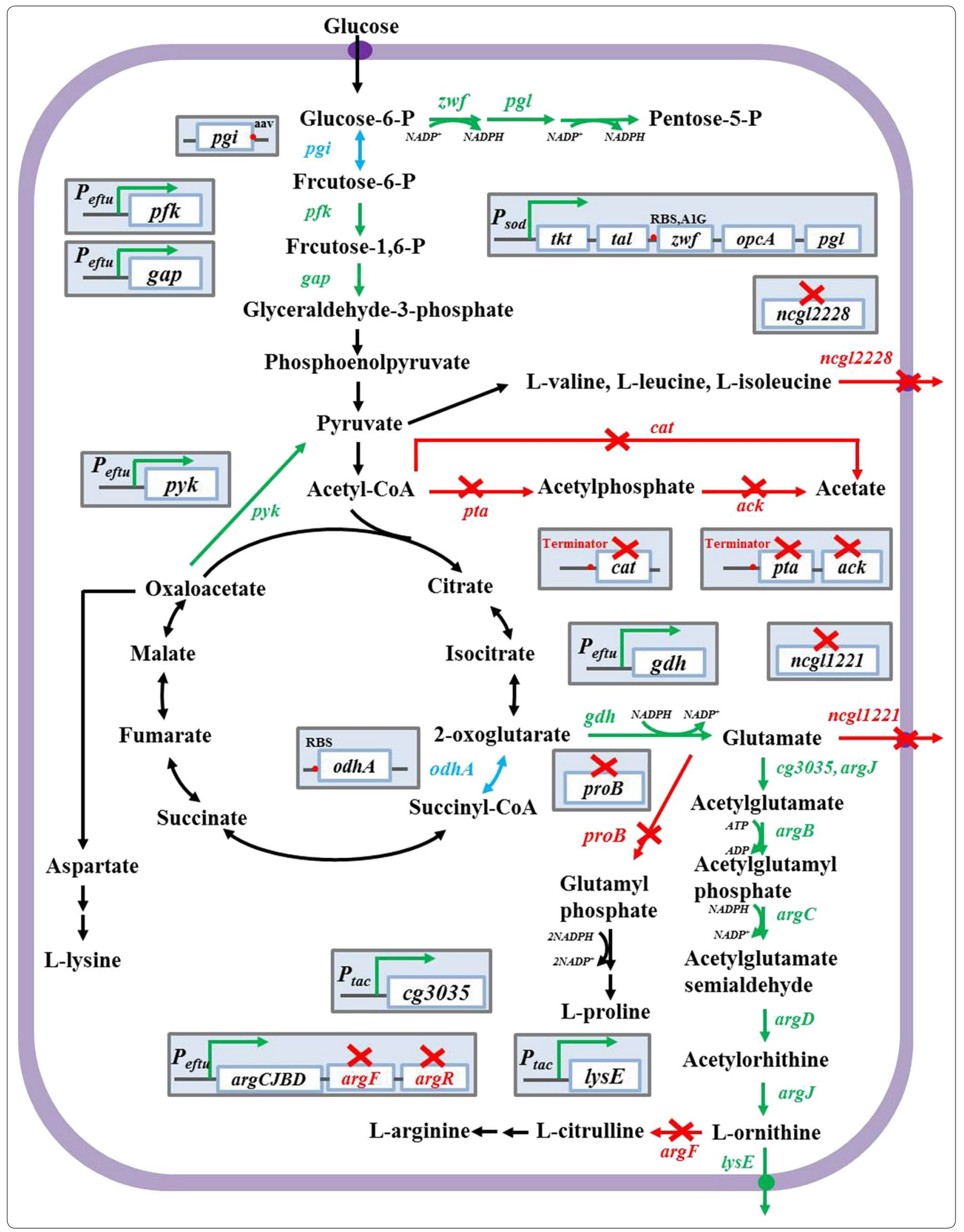


Table 1 Strains and plasmids used in this study

\begin{tabular}{|c|c|c|}
\hline Strain/plasmid & Characteristic & Source \\
\hline \multicolumn{3}{|l|}{ Strain } \\
\hline E. coli DH5a & Clone host strain & Transgen \\
\hline $\mathrm{SO} 1$ & $\begin{array}{l}\text { C. glutamicum S9114 with deletion of argF, ncgl1221, argR, putP, attenuation of odhA, proB, ncgl2228, overexpression of lysE, } \\
\text { gdh, argCJBD }\end{array}$ & Lab stock \\
\hline $\mathrm{SO} 2$ & SO1 carrying expression vector pEC-XK99E & This study \\
\hline $\mathrm{SO} 3$ & SO1 carrying expression vector pEC-cg3035 & This study \\
\hline $\mathrm{SO} 4$ & SO1 with $P_{\text {tac }}$ promoter inserted in front of cg3035 & This study \\
\hline SO5 & SO1 with terminator inserted in front of pta & This study \\
\hline SO6 & SO1 with terminator inserted in front of cat & This study \\
\hline SO7 & SO1 with $P_{\text {eftu }}$ promoter inserted in front of $p f k A$ & This study \\
\hline SO8 & SO1 with $\mathrm{P}_{\text {eftu }}$ promoter inserted in front of gap & This study \\
\hline SO9 & SO1 with $\mathrm{P}_{\text {eftu }}$ promoter inserted in front of pyk & This study \\
\hline SO10 & SO1 with $\mathrm{P}_{\text {effu }}$ promoter inserted in front of pfkA and $\mathrm{P}_{\text {tac }}$ promoter inserted in front of cg3035 & This study \\
\hline SO11 & $\begin{array}{l}\text { SO1 with } \mathrm{P}_{\text {effu }} \text { promoter inserted in front of pfkA, } \mathrm{P}_{\text {tac }} \text { promoter inserted in front of cg3035 and a terminator inserted in } \\
\text { front of pta }\end{array}$ & This study \\
\hline SO12 & $\begin{array}{l}\text { SO1 with } \mathrm{P}_{\text {eftu }} \text { promoter inserted in front of pfkA, } \mathrm{P}_{\text {tac }} \text { promoter inserted in front of cg3035 and a terminator inserted in } \\
\text { front of cat }\end{array}$ & This study \\
\hline SO13 & $\begin{array}{l}\text { SO1 with } \mathrm{P}_{\text {effu }} \text { promoter inserted in front of pfkA, } \mathrm{P}_{\text {tac }} \text { promoter inserted in front of cg3035 and terminator inserted in } \\
\text { front of pta and cat }\end{array}$ & This study \\
\hline SO14 & SO12 with an AAV degradation label inserted in the $C$ terminal of pgi & This study \\
\hline SO15 & SO14 with synthetic RBS and A1G replacement in front of zwf. & This study \\
\hline SO16 & SO15 with $P_{\text {tac }}$ promoter inserted in front of tkt operon. & This study \\
\hline \multicolumn{3}{|l|}{ Plasmid } \\
\hline pK18mobsacB & Mobilizable vector, allows for selection of double crossover in C. glutamicum, $\mathrm{Km}^{\mathrm{R}}$, sacB & {$[16]$} \\
\hline pEC-XK99E & A shuttle expression vector, $\mathrm{Km}^{\mathrm{R}}$ & Lab stock \\
\hline pEC-cg3035 & A derivative of pEC-XK99E, harboring cg3035 gene from C. glutamicum S9114 under its native promoter & This study \\
\hline pK18- $P_{\text {tac }}-\operatorname{cg} 3035$ & A derivative of pK18mobsacB, harboring $P_{\text {tac }}$-cg3035 fragment & This study \\
\hline pK18-T-pta & A derivative of pK18mobsacB, harboring T-pta fragment & This study \\
\hline pK18-T-cat & A derivative of pK18mobsacB, harboring T-cat fragment & This study \\
\hline pK18-P $\mathrm{P}_{\text {eftu }}-\mathrm{pfkA}$ & A derivative of pK18mobsacB, harboring $P_{\text {eftu }}$-pfkA fragment & This study \\
\hline pK18- $\mathrm{P}_{\text {eftu-gap }}$ & A derivative of pK18mobsacB, harboring $\mathrm{P}_{\text {eftu }}$-gap fragment & This study \\
\hline pK18- $P_{\text {eftu }}-p y k$ & A derivative of pK18mobsacB, harboring $\mathrm{P}_{\text {eftu }}$-pyk fragment & This study \\
\hline pK18-pgi-aav & A derivative of pK18mobsacB, harboring pgi-AAV fragment & This study \\
\hline pK18-SB-zwf & A derivative of pK18mobsacB, harboring SB-zwf fragment & This study \\
\hline pK18- $P_{\text {tac }}-t k t$ & A derivative of $\mathrm{pK} 18$ mobsacB, harboring $\mathrm{P}_{\text {tac }}{ }^{-t k t}$ fragment & This study \\
\hline
\end{tabular}

Superscript " $\mathrm{R}$ " indicates resistance to the following antibiotics: $K m$ kanamycin

into the expression vector pEC-XK99E by Gibson assembly to construct a constitute expression cassette pECcg3035. After isolation from E. coli, this recombinant plasmid and empty vector $\mathrm{pEC-XK99E}$ were transformed into $\mathrm{SO} 1$ by electroporation. The positive transformants were pointed out and confirmed by colony PCR.

For $\mathrm{P}_{t a c}$ promoter insertion in front of $\mathrm{cg} 3035$, the upstream region and the coding region of cg3035 was amplified. A strong $\mathrm{P}_{t a c}$ promoter was introduced between the upstream region and coding region by primers. The overlapping fragment containing the upstream region, $\mathrm{P}_{t a c}$ promoter, and the coding region was cloned into the Hind III/Xba I sites in the suicide vector pK18mobsacB by Gibson assembly. The engineered plasmid was then transferred into strain SO1 by electroporation. After double crossover recombination, the positive recombinant strain was detected by colony PCR. Similarly, the insertion of the $\mathrm{P}_{\text {eftu }}$ promoter in front of $p f k A$, gap and pyk or insertion of $\mathrm{P}_{t a c}$ in front of $t k t$ was also performed using these procedures.

To attenuate the expression of genes, strategy described in our previous study were carried out [18]. To attenuate the expression of $p t a$ and cat, a transcription terminator was introduced into the right upstream of the genes 
pta and cat which lines between the upstream fragment and downstream fragment by PCR. The recombinant fragment was then cloned into the Hind III/Xba I sites in the suicide vector pK18mobsacB by Gibson assembly. After extraction from $E$. coli, this engineered vector was transformed into SO1 by electroporation. After double crossover recombination, the mutant strains with a terminator inserted in the upstream region of $p t a$ and cat were determined by colony PCR. When constructing the mutant strains, an AAV taq was inserted in the C terminal of $p g i$ with a synthetic RBS and G1A replacement in $z w f$. The RBS sequence with the predicted translation start strength of 50000 au was designed using the RBS Calculator (https://www.denovodna.com/software/ doLogin) and is listed in Additional file 1: Table S2.

\section{Fermentation in shake flasks}

Fermentation with recombinant strains was performed in batch culture in shake flasks as described in our previous work [14, 15]. A single clone of the mutants was activated on LB agar plate for two cycles of $12 \mathrm{~h}$. Subsequently, a ring of bacteria was inoculated into $10 \mathrm{~mL}$ of seed medium in a $100-\mathrm{mL}$ normal shake flask. The seed medium consisted of (per liter) $25 \mathrm{~g}$ glucose, $10 \mathrm{~g}$ yeast extract, $10 \mathrm{~g}$ corn steep liquor, $15 \mathrm{~g}\left(\mathrm{NH}_{4}\right)_{2} \mathrm{SO}_{4}$, $2.5 \mathrm{~g} \mathrm{MgSO}_{4} \cdot 7 \mathrm{H}_{2} \mathrm{O}, 1 \mathrm{~g} \mathrm{KH}_{2} \mathrm{PO}_{4}, 0.5 \mathrm{~g} \mathrm{~K}_{2} \mathrm{HPO}_{4}, 0.5 \mathrm{~g}$ $\mathrm{Na}_{2} \mathrm{HPO}_{4}$, and $10 \mathrm{~g} \mathrm{CaCO}_{3}$. After $11 \mathrm{~h}$ of cultivation at $32{ }^{\circ} \mathrm{C}$ and $220 \mathrm{rpm}$, the appropriate amount of culture was transferred to $24 \mathrm{~mL}$ of fermentation medium in a 250 -mL baffle shake flask. Initial $\mathrm{OD}_{600}$ of the fermentation culture was adjusted to one. The fermentation medium consisted of (per liter) $100.0 \mathrm{~g}$ glucose, $20.0 \mathrm{~g}$ corn steep liquor, $50.0 \mathrm{~g}\left(\mathrm{NH}_{4}\right)_{2} \mathrm{SO}_{4}, 2.5 \mathrm{~g} \mathrm{MgSO}_{4} \cdot 7 \mathrm{H}_{2} \mathrm{O}$, $1.0 \mathrm{~g} \mathrm{KH}_{2} \mathrm{PO}_{4}, 0.5 \mathrm{~g} \mathrm{~K}_{2} \mathrm{HPO}_{4}, 0.5 \mathrm{~g} \mathrm{Na}_{2} \mathrm{HPO}_{4}, 0.02 \mathrm{~g}$ $\mathrm{FeSO}_{4} \cdot 7 \mathrm{H}_{2} \mathrm{O}, 0.02 \mathrm{~g} \mathrm{MnSO}_{4} \cdot 4 \mathrm{H}_{2} \mathrm{O}$, and $10 \mathrm{~g} \mathrm{CaCO}_{3}$. The initial $\mathrm{pH}$ was adjusted to 7.0. All cultures were grown at $32{ }^{\circ} \mathrm{C}$ and $250 \mathrm{rpm}$, and $200-\mu \mathrm{L}$ samples were collected every $12 \mathrm{~h}$ to measure L-ornithine concentration, cell density, and residual glucose concentration. If necessary, $50 \mathrm{mg} / \mathrm{L}$ kanamycin was used to cultivate $E$. coli and $12.5 \mathrm{mg} / \mathrm{L}$ kanamycin was used to cultivate $C$. glutamicum.

\section{Measurement of NAGS enzyme activity}

For NAGS enzyme activity analysis, $20 \mathrm{~mL}$ of fermentation samples were collected at $10 \mathrm{~h}$ by centrifugation (at $5000 \mathrm{rpm}, 4{ }^{\circ} \mathrm{C}$, and $10 \mathrm{~min}$ ) and washed twice with $100 \mathrm{mM}$ Tris- $\mathrm{HCl}$ (pH 7.5) which was supplemented with $20 \mu \mathrm{M}$ PMSF. Following the pure cells were incubated in $5 \mathrm{~mL}$ of $100 \mathrm{mM}$ Tris- $\mathrm{HCl}(\mathrm{pH} 7.5)$ containing $30 \%(\mathrm{v} / \mathrm{v})$ glycerol and $10 \mathrm{mg} / \mathrm{mL}$ lysozyme at $37{ }^{\circ} \mathrm{C}$ for $3 \mathrm{~h}$ and then disrupted by sonication. After removing cell debris by centrifugation, the supernatant was collected as crude enzyme, and the protein concentration was determined by bicinchoninic acid (BCA) assay using bovine serum albumin as the standard. NAGS specific activity assay was performed as described previously [19].

\section{RT-PCR}

For RNA analysis, $500 \mu \mathrm{L}$ of fermentation samples were collected at $12 \mathrm{~h}$. RNA extraction and RT-PCR assays were performed as described in our early report [20].

\section{Measurement of glucose consumption, optical density, and metabolite analysis}

After dissolving $\mathrm{CaCO}_{3}$ in $0.125 \mathrm{~mol} / \mathrm{L} \mathrm{HCl}$, cell growth was monitored by measuring the $\mathrm{OD}_{600}$ using a microplate reader (BioTek Instruments, Winooski, VT, USA). L-Ornithine concentrations were determined by colorimetry using ninhydrin, as described previously [21, 22]. L-Ornithine standard curve of colorimetric assay was listed in Additional file 1: Figure S1. The fermentation supernatant was passed through a $0.22-\mu \mathrm{m}$ filter and analyzed for glucose levels, using a SBA-40C biosensor (developed by Biology Institute of Shandong Academy of Sciences). Acetic acid were analyzed as described previously [23]. All experiments were conducted in triplicate; the data were averaged and presented as mean \pm standard deviation (SD).

\section{Results \\ Overexpression of $\mathrm{cg} 3035$ exerts a positive effect on $\mathrm{L}$-ornithine production}

Glutamate acetylation is the first step in the conversion of glutamate to L-ornithine and prevents glutamate from cyclisation and further conversion to L-proline. Petri et al. reported that glutamate acetylation is catalyzed by $c g 3035$, which updated the previous knowledge that $\operatorname{argJ}$ encodes the bifunctional enzyme for glutamate acetylation and $N$-acetylornithine deacetylation [19]. In a previous study, we constructed a high L-ornithine producing strain C. glutamicum strain SO1 (S9114 with deletion of $\operatorname{argF}, n c g l 1221, \operatorname{argR}$, and $p u t P$, attenuation of $o d h A, p r o B$, and $n c g l 2228$, and overexpression of lysE, $g d h$, and $\operatorname{argCJBD}$ ) and found that $\operatorname{argCJBD}$ expression was not the rate-limiting step for L-ornithine accumulation. Therefore, glutamate acetylation prevented further improvement of L-ornithine accumulation. To overcome this barrier, cg3035 was overexpressed using an expression vector PEC-XK99E in the engineered strain SO1, thus generating strain SO3. Compared with strain SO1, NAGS enzyme activity in strain $\mathrm{SO} 3$ was improved by 5.63-fold, which suggested that cg3035 was successful overexpressed. The empty plasmid pEC-XK99E was also transformed into strain $\mathrm{SO} 1$ to produce strain $\mathrm{SO} 2$ as a control. To fully evaluate the performance of the 
engineered strains $\mathrm{SO} 2$ and $\mathrm{SO} 3$, shake flask fermentation was performed. After $72 \mathrm{~h}$ of cultivation, the mutant strain SO3 produced $26.2 \mathrm{~g} / \mathrm{L}$ of L-ornithine, which is $16.5 \%$ higher than that $(22.5 \mathrm{~g} / \mathrm{L})$ obtained with strain $\mathrm{SO} 2$ (Fig. 2a). The cell growth of strain SO3 as well as its glucose consumption was comparable to that of the control strain SO2; indicating cg3035 overexpression is nontoxic to C. glutamicum (Fig. 2b). These results confirmed our speculation that glutamate acetylation plays an important role in L-ornithine biosynthesis. Inspired by the results of plasmid-based cg3035 overexpression, a strong mutant $\mathrm{P}_{t a c}$ promoter developed by Jakob [24] was integrated into the chromosome of the parent strain SO1 to address the challenges associated with genetic instability using a vector, to generate strain SO4. This strategy was successfully applied fsor overexpressing lysE during our previous work. The relative mRNA level of $c g 3035$ and NAGS enzyme activity in strain SO4 were 14.7-fold and 3.62-fold higher than that in the control strain, respectively, which illustrated that the expression of $c g 3035$ was effectively strengthened through the insertion of $\mathrm{P}_{t a c}$ promoter (Fig. 2c and Table 2). During $72 \mathrm{~h}$ of fermentation, the L-ornithine production titer of strain SO4 was measured to be $26.8 \mathrm{~g} / \mathrm{L}$, which was increased by $12.6 \%$ compared to that of the parent strain SO1 (23.8 g/L) (Fig. 2d). Similar to the result obtained by using a vector for $c g 3035$ overexpression, the engineered strain SO4 exhibited coincident growth and glucose consumption as the control strain SO1 (Fig. 2e, f). In consequence, these results provide detailed knowledge of cg3035 expression to improve L-ornithine accumulation.

\section{Improvement of L-ornithine production by attenuating the acetate synthesis pathway}

Glutamate acetylation, a confirmed rate-limiting step for further improvement of L-ornithine production in engineered C. glutamicum SO1, is the first reaction in the L-ornithine pathway that uses acetyl-CoA as second substrate next to glutamate. We speculated that insufficient acetyl-CoA supplementation hindered the biosynthesis of $\mathrm{L}$-ornithine in these strains. To spare acetyl-CoA for L-ornithine biosynthesis, we considered acetic acid biosynthesis that consumes acetyl-CoA and consists of two synthetic branches catalyzed by the products of $p t a$ and $a c k$, or cat. Therefore, we attenuated these two acetate synthetic branches by insertion of a terminator, which was used to attenuate the expression of proB and $n c g l 2228$ in previous work, in the upstream region of these genes, thus generating strains $\mathrm{SO} 5$ and SO6. Considering that $p t a$ and ack are listed as an operon, ack expression will also be attenuated by inserting a terminator in the upstream region of pta. The performance of these two strains was evaluated by shake flask fermentation. As shown in Fig. 3a, b, the expression of pta and ack in the mutant strain $\mathrm{SO} 5$ and the relative mRNA level of cat in the engineered strain SO6 was significantly reduced compared with that in the parental strain SO1. In addition, the yield of acetic acid produced by strain $\mathrm{SO} 5$ and SO6 were also reduced approximately 2.5 -fold than strain SO1 (Table 2). This indicated that insertion of a terminator upstream of pta and cat was an efficient strategy to downregulate their expression. Strain SO5 and SO6 produced 25.1 and $26 \mathrm{~g} / \mathrm{L}$ of L-ornithine, which was 5.5 and 9.1\% higher than that of the control strain SO1 $(23.8 \mathrm{~g} / \mathrm{L})$ (Fig. 3c). Results obtained per $12 \mathrm{~h}$ represent a significant improvement of L-ornithine synthesis compared with that of the control strain. Despite altered acetate biosynthesis, the engineered strains $\mathrm{SO} 5$ and $\mathrm{SO} 6$ grow robustly in the fermentation medium, maintaining a stable rate of glucose consumption (Fig. 3d). These results indicate that attenuating the expression of genes involved in acetate biosynthesis is a viable strategy for improving L-ornithine accumulation.

\section{Effect of overexpressing enzymes of the glycolytic pathway on L-ornithine production}

As previously demonstrated, plasmid-based overexpression of gap encoding NAD-dependent glyceraldehyde-3-phosphate dehydrogenase, $p f k A$ encoding 6-phosphofructokinase, and pyk encoding pyruvate kinase was able to improve L-ornithine production in the engineered C. glutamicum ATCC 13032 strain [22]. Therefore, to examine if enhancing the expression of these genes in strain SO1 could further improve L-ornithine production, we inserted a strong $\mathrm{P}_{\text {eftu }}$ promoter into the upstream region of $p f k A$, gap, and $p y k$ in strain SO1, to construct strains $\mathrm{SO}$, $\mathrm{SO}$, and $\mathrm{SO}$, respectively. After cultivation in the fermentation media for about $72 \mathrm{~h}$, strains $\mathrm{SO} 7, \mathrm{SO}$, and $\mathrm{SO} 9$ produced 26.5, 22.8 , and $21.5 \mathrm{~g} / \mathrm{L}$ of L-ornithine (Fig. 4a). The yield of L-ornithine produced by strain $\mathrm{SO} 7$ with $p f k A$ overexpression was $11.2 \%$ higher than that of the parental strain SO1. Strain SO8 with gap overexpression produced the same amount of L-ornithine as that of the parent strain SO1. The yield of L-ornithine produced by strain SO9 with $\mathrm{P}_{\text {eftu }}$ inserted in front of $p y k$ was decreased by 9.7\% compared to strain SO1. The growth of strain SO8 and $\mathrm{SO} 9$ was slower than that of the engineered strains $\mathrm{SO} 1$ and SO7. Glucose consumption by strain $\mathrm{SO} 7$ was slightly faster than that by strains $\mathrm{SO}, \mathrm{SO} 8$, and $\mathrm{SO}$. Taken together, those results suggest that modulation of $p f k A$ promotes $\mathrm{L}$-ornithine accumulation in engineered C. glutamicum S9114. 

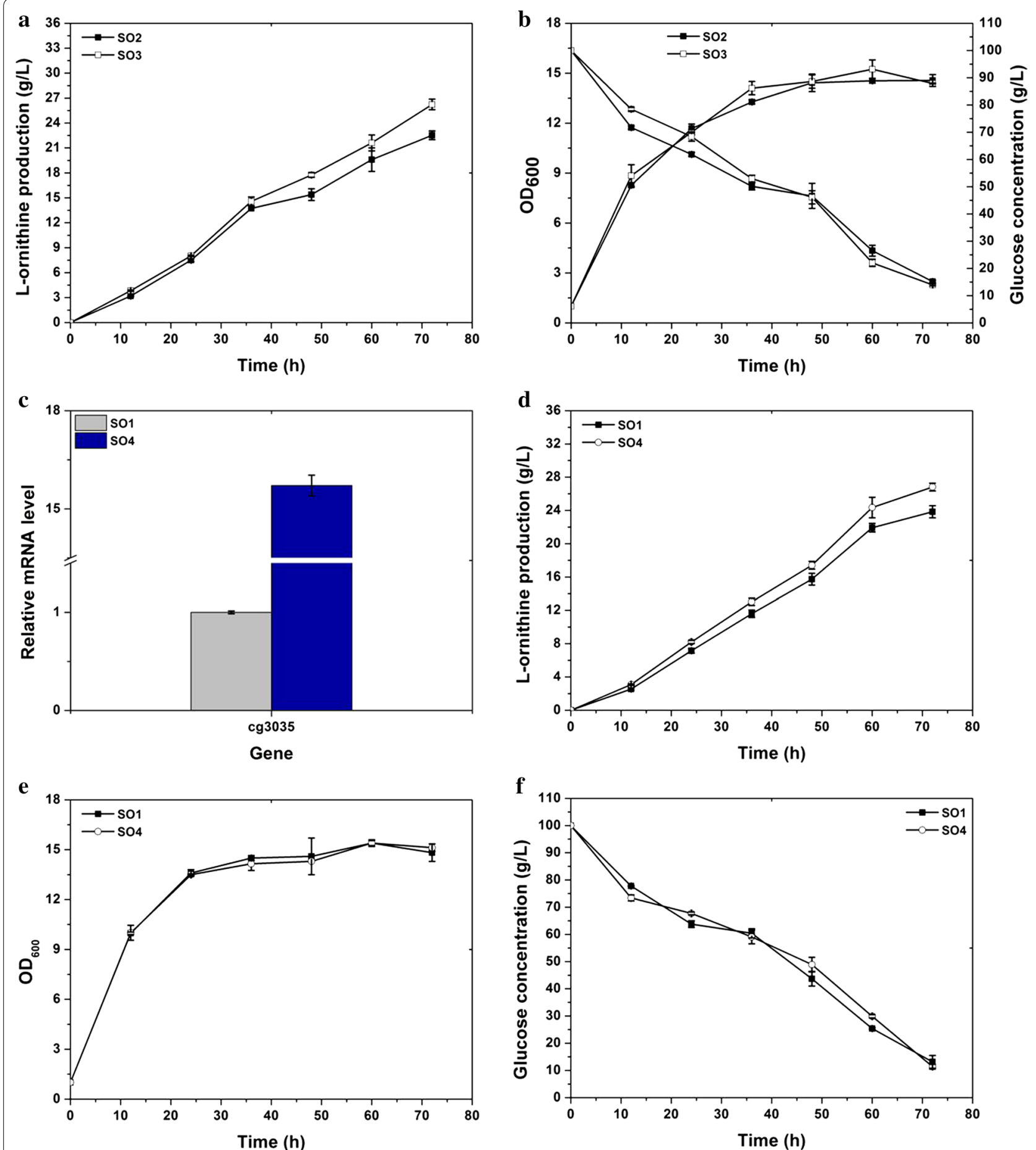

Fig. 2 Improvement of L-ornithine production by cg3035 overexpression. a L-Ornithine production curves for strains $\mathrm{SO} 2$ (SO1 carrying pEC-XK99E) and SO3 (SO1 carrying pEC-cg3035). b Cell growth and glucose consumption of strains SO2 and SO3. c Relative transcription level of cg3035 in engineered strains SO1 and SO4 (SO1 with $\mathrm{P}_{\text {tac }}$ promoter inserted upstream of cg3035). $\mathbf{d}$ L-Ornithine production curves for the engineered strains $\mathrm{SO} 1$ and $\mathrm{SO} 4$. e Cell growth. f Glucose consumption. Samples were collected per $12 \mathrm{~h}$ for determining fermentation parameters. Results of standard deviations present in three individual experiments 
Table 2 Acetic acid concentration in $72 \mathrm{~h}$ fermentation broth

\begin{tabular}{lll}
\hline Strains & $\begin{array}{l}\text { Relative NAGS specific } \\
\text { activity }\end{array}$ & $\begin{array}{l}\text { Acetic acid } \\
\text { concentration } \\
\text { (g/L) }\end{array}$ \\
\hline $\mathrm{SO} 1$ & $1 \pm 0.13$ & $3.85 \pm 0.14$ \\
$\mathrm{SO} 3$ & $6.63 \pm 0.07$ & - \\
$\mathrm{SO} 4$ & $4.62 \pm 0.21$ & - \\
$\mathrm{SO} 5$ & - & $1.05 \pm 0.05$ \\
$\mathrm{SO} 6$ & - & $1.34 \pm 0.06$ \\
\hline
\end{tabular}

Combination of $P_{\text {tac }}-c g 3035, P_{\text {eftu }}-$ pfkA, T-pta, and T-cat targets and its influence on $\mathrm{L}$-ornithine production From the above results, several efforts including independently inserting a $\mathrm{P}_{t a c}$ in front of $c g 3035$, inserting a $\mathrm{P}_{\text {eftu }}$ in front of $p f k A$, and inserting a terminator in the upstream region of $p t a$ or cat were observed to enhance L-ornithine accumulation. Since our major goal is to construct a high L-ornithine producing strain, we combined these targets and constructed strain $\mathrm{SO} 10$ carrying $P_{t a c}-c g 3035$ and $P_{e f t u}-p f k A$, SO11 with $P_{t a c}{ }^{-}$ cg3035, $P_{\text {eftu }}-p f k A$, and T-cat, SO12 with $P_{t a c}-c g 3035$, $P_{\text {eftu }}-p f k A$, and T-pta, and SO13 with $P_{t a c}-c g 3035, P_{\text {eftu }}{ }^{-}$ pfkA, T-pta, and T-cat. Next, the effects of these modifications on L-ornithine accumulation were tested in the context of shake flask fermentation. As shown in Fig. 5a, mutant strains incorporating the modulation of these targets produced more L-ornithine compared to the parent strain SO1. The highest L-ornithine production titer in $72 \mathrm{~h}$ cultivation was produced by strain SO12 and reached $29 \mathrm{~g} / \mathrm{L}$, which was $21.8 \%$ higher than that obtained with strain SO1 (23.8 g/L). Unexpectedly,
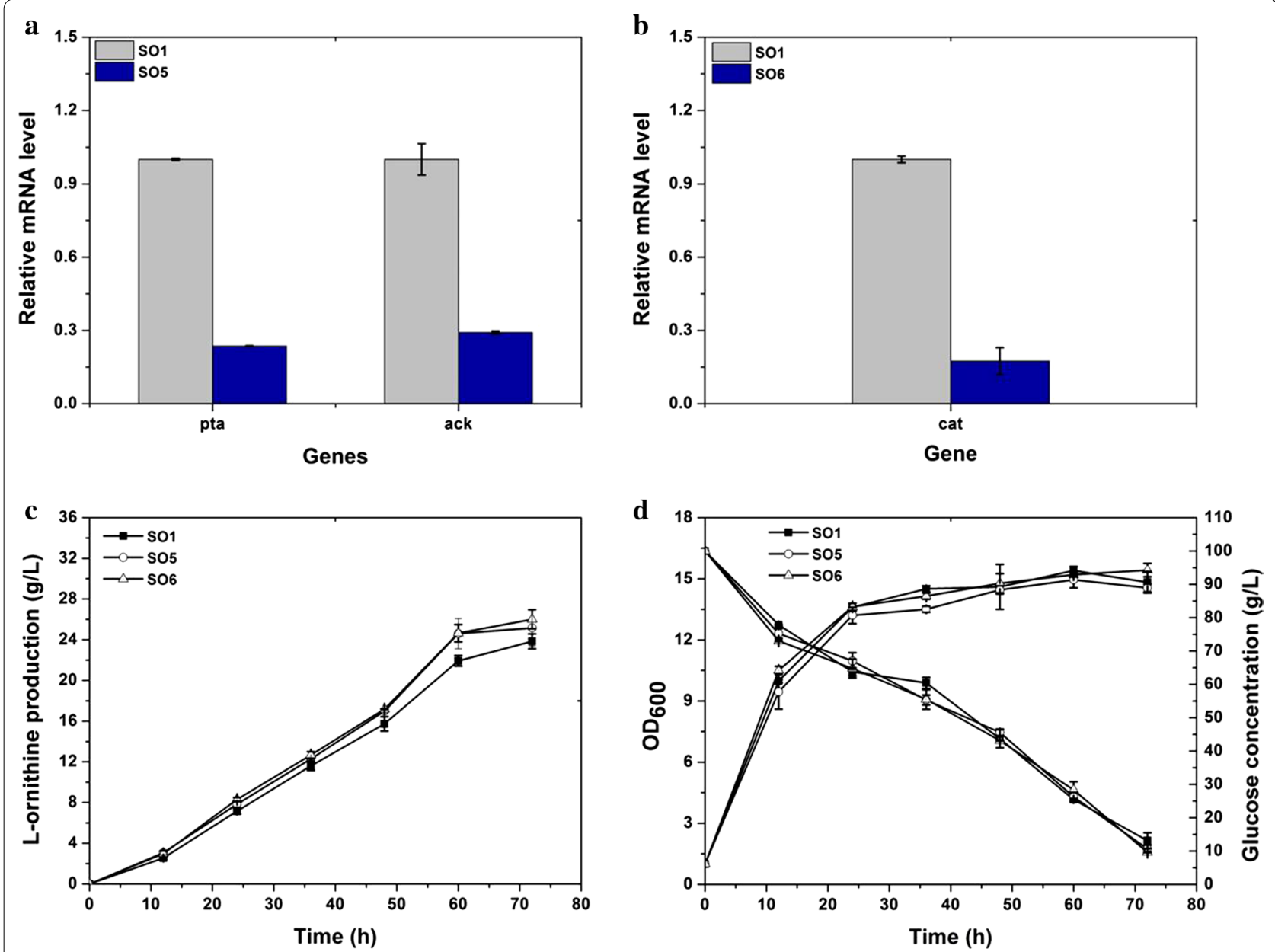

Fig. 3 Blocking acetate biosynthesis by inserting a terminator into the upstream region of pta and cat, and its effect on $\mathbf{L}$-ornithine production. a Relative pta and ack mRNA levels in SO1 and SO5 (SO1 carrying modulation in pta). Samples at the $12 \mathrm{~h}$ time point were subjected to transcript analysis during fermentation cultivation. b Relative cat mRNA levels in $\mathrm{SO} 1$ and $\mathrm{SO} 6$ (SO1 carrying modulation in cat). c L-Ornithine production by the engineered strains SO1, SO5, and SO6. $\mathbf{d}$ Cell growth and glucose consumption. Samples were collected per $12 \mathrm{~h}$ for determining the fermentation parameters. Results of standard deviations present in three individual experiments 

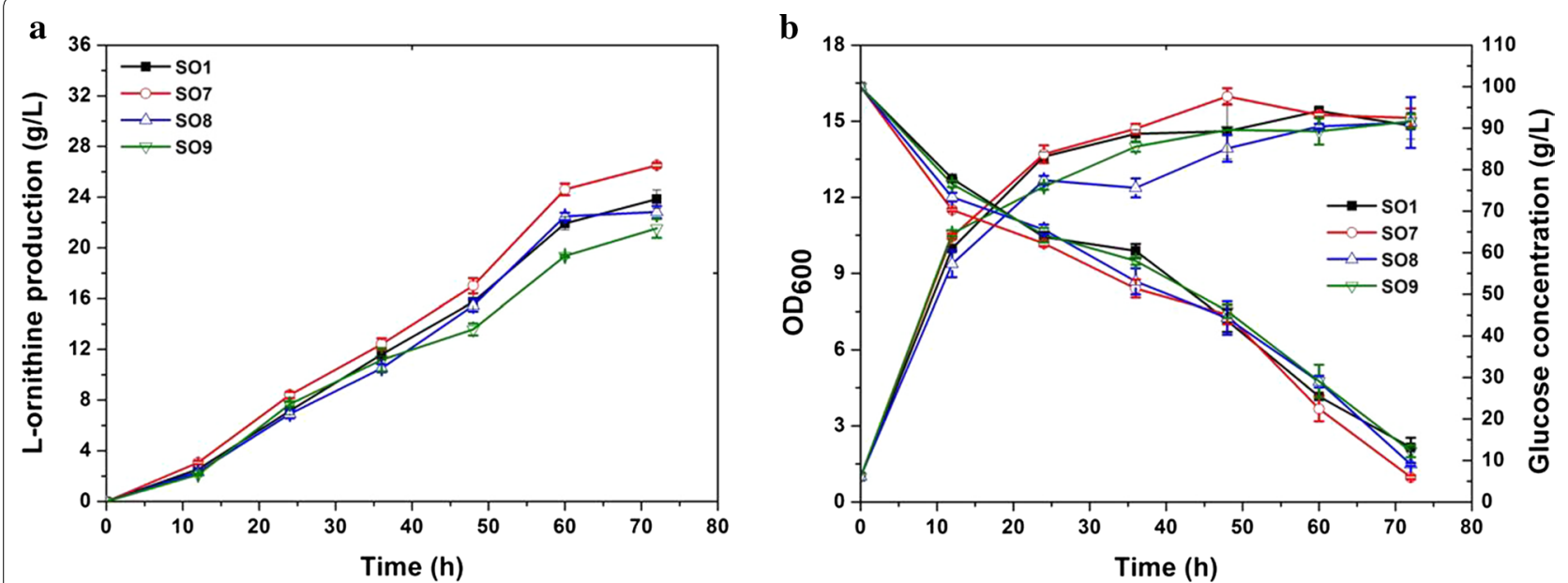

Fig. 4 Assessment of L-ornithine productivity and other characterizations of strains $\mathrm{SO}$ 1, SO7 (SO1 carrying $\mathrm{P}_{\text {eftu }}$ promoter inserted upstream of pfkA), SO8 (SO1 with $\mathrm{P}_{\text {eftu }}$ promoter inserted upstream of gap), and SO9 (SO1 with $\mathrm{P}_{\text {eftu }}$ promoter inserted upstream of pyk). a L-Ornithine production curves for strains SO1, SO7, SO8, and SO9. b Cell growth and glucose consumption. Samples were collected per $12 \mathrm{~h}$ for fermentation parameter determination. Data represent average values and standard deviations from three individual experiments
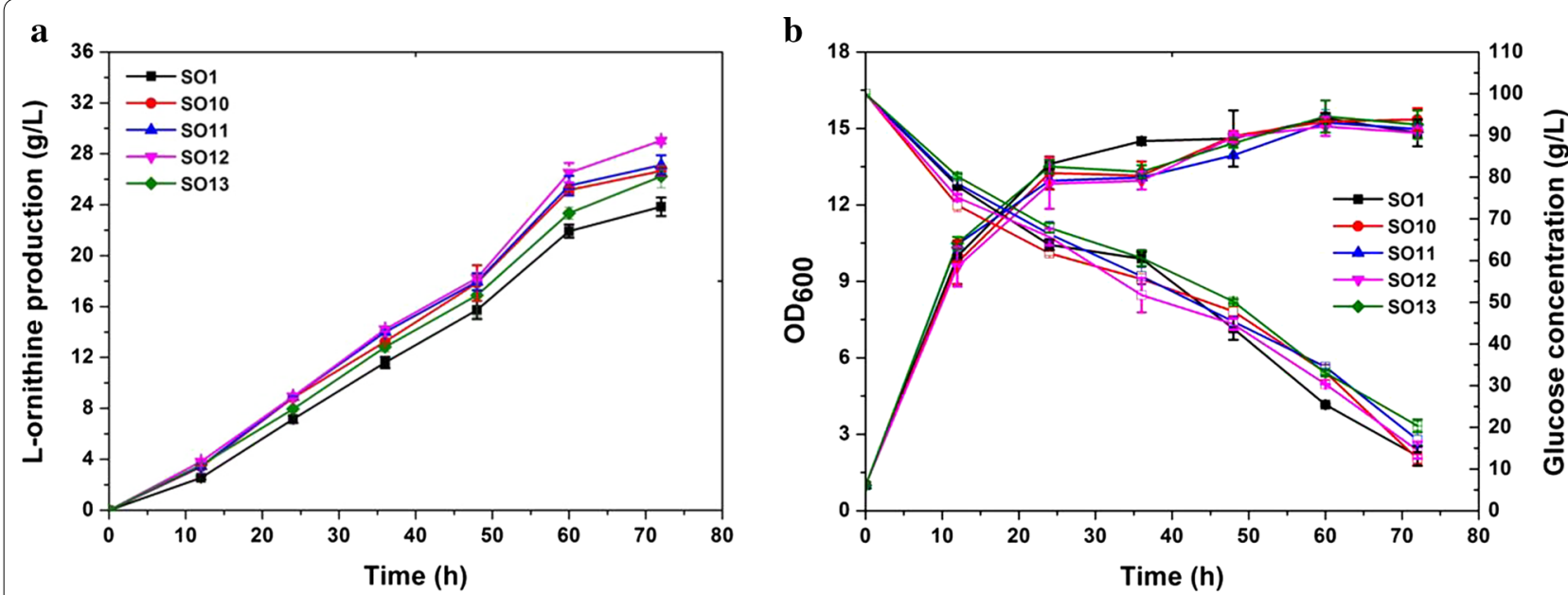

Fig. 5 L-Ornithine productivity, cell growth, and glucose consumption during cultivation of SO1, SO10 (SO1 carrying $\mathrm{P}_{\text {effu }}$ promoter inserted upstream of pfkA and $P_{\text {tac }}$ promoter inserted upstream of cg3035), SO11 (SO1 carrying $P_{\text {effu }}$ promoter inserted upstream of pfkA, $P_{\text {tac }}$ promoter inserted upstream of cg3035 and terminator inserted in the upstream region of cat), SO12 (SO1 carrying the $P_{\text {eftu }}$ promoter inserted upstream of pfk $A, P_{\text {tac }}$ promoter inserted upstream of cg3035, and a terminator inserted upstream region of pta), and SO13 (SO1 carrying the $P_{\text {eftu }}$ promoter inserted upstream of $p f k A, P_{\text {tac }}$ promoter inserted upstream of cg3035, and a terminator inserted in the upstream region of pta and cat). a L-Ornithine production curves for strains SO1 (black square), SO10 (red circle), SO11 (blue upper triangle), SO12 (pink lower triangle), and SO13 (green quadrangle). b Cell growth and glucose consumption. Samples were collected per $12 \mathrm{~h}$ for fermentation parameter determination. Data represent the average values and standard deviations from three individual experiments

strain SO13, which possessed all of these modifications, exhibited a lower L-ornithine production performance and relatively slow glucose utilization efficiency compared to strain SO12 (Fig. 5b). In conclusion, these results illustrate that these targets exhibit a positive synergistic effect on improving L-ornithine production.

\section{Enhancement of $\mathrm{L}$-ornithine production by redirecting} the metabolic flux into pentose phosphate pathway

L-Ornithine biosynthesis in C. glutamicum requires 2 mol of NADPH. Improving NADPH supplementation is an important factor for L-ornithine accumulation. Several strategies such as redirecting carbon flux to the pentose phosphate pathway, overexpression of 
Clostridium acetobutylicum gapC, deletion of putative oxidoreductases, and so on for enhancing NADPH supplementation were confirmed to increase the production titer of L-ornithine. Nevertheless, as NADPH supply is frequently described as a limiting factor for L-ornithine biosynthesis and the engineered strain $\mathrm{SO} 13$ does not carry any genetic modification to enhance NADPH supply, the question of whether NADPH supply is limiting or not remains open. Therefore, we redirected the carbon flux to the pentose phosphate pathway through attenuation of $p g i$, overexpression of the $t k t$ operon, and changing the RBS and translation start codon of $z w f$ in the engineered strain SO12, to generate strains SO14 (SO12 with an AAV taq inserted in the C-steam of pgi),
SO15 (SO14 with a strong $\mathrm{P}_{t a c}$ promoter inserted in front of the $t k t$ operon), and SO16 (SO15 with G1A and RBS replacement in $z w f)$. The relative $t k t$ and $z w f$ mRNA levels in strain SO16 were approximately 1.5-fold higher than those in strain SO15, indicating that insertion of the $\mathrm{P}_{\text {tac }}$ promoter is able to improve the transcription level of the $t k t$ operon (Fig. 6a). Fermentation in a shake flask revealed that strains SO14, SO15, and SO16 produced $30.38,32.30$, and $32.38 \mathrm{~g} / \mathrm{L}$ L-ornithine with productivities of $0.359,0.389$, and $0.395 \mathrm{~g} / \mathrm{g}$ glucose, respectively, which is $4.5,11.4$, and $11.4 \%$ higher than that of strain SO12 (29 g/L). Compared with strain SO12, SO14, and SO15, strain SO16 exhibited a slightly lower growth (Fig. 6c). Strain SO14 demonstrated relatively lower
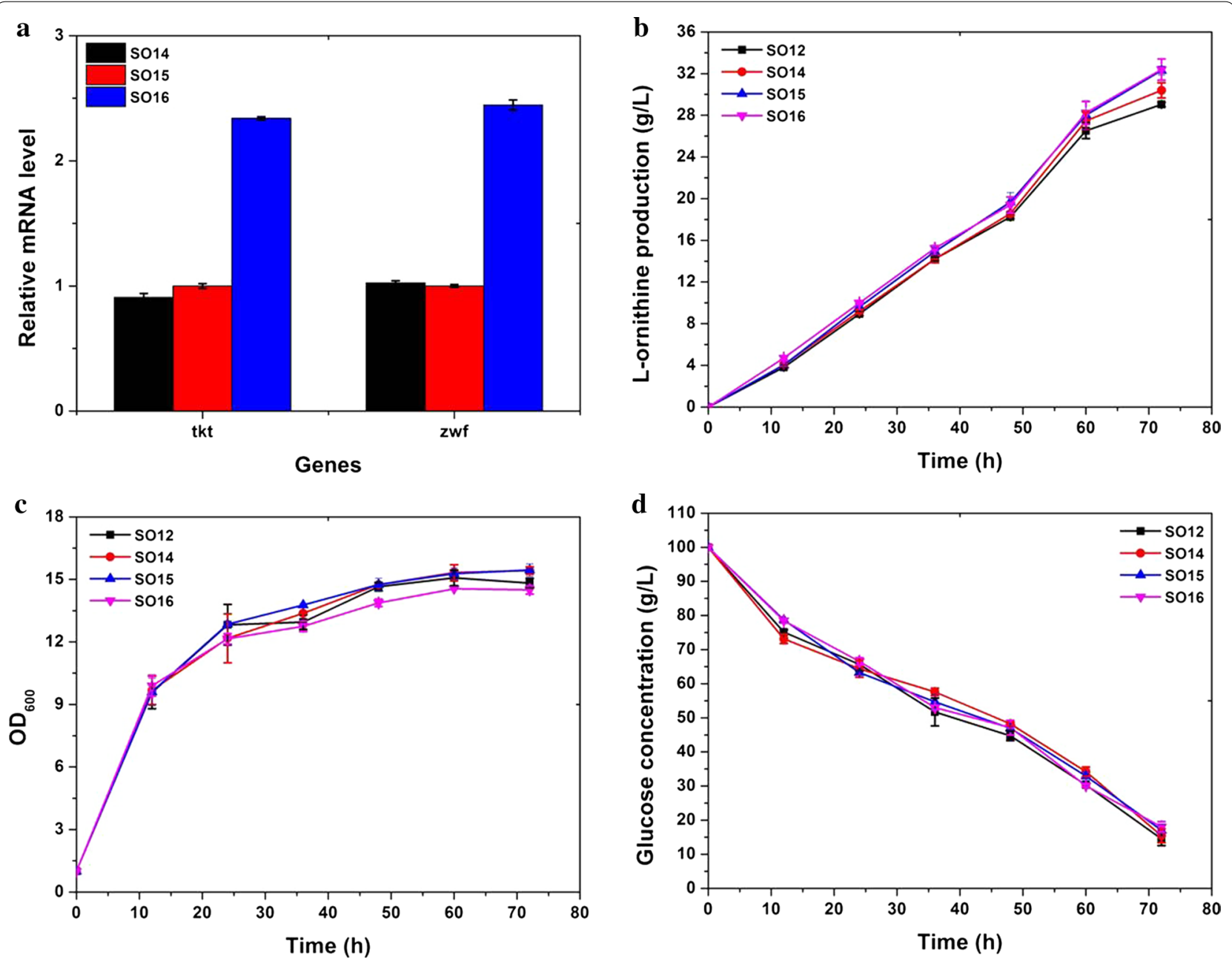

Fig. 6 Pentose phosphate pathway modulation and its effect on L-Ornithine production. a Relative tkt and zwf mRNA levels in strains SO15 (SO12 carrying AAV taq inserted into the C-terminal of Pgi and RBS together with start codon T1A replacement in front of zwf) and SO16 (SO15 carrying $\mathrm{P}_{\text {tac }}$ promoter inserted in front of $t k t$ operon). Samples at the $12 \mathrm{~h}$ time point were subjected to transcript analysis during fermentation cultivation. b L-Ornithine production curves for strains SO12 (black square), SO14 (SO12 carrying AAV taq inserted into the C-terminal of Pgi) (red cycle), SO15 (blue upper triangle), and SO16 (pink lower triangle). c Growth profiles of these strains. d Glucose consumption curves. Samples were collected per $12 \mathrm{~h}$ for fermentation parameter determination. Data represent the average values and standard deviations from three individual experiments 
glucose consumption compared to other strains (Fig. 6d). These results illustrate that improving the availability of NADPH is an inevitable modulation for L-ornithine accumulation in C. glutamicum.

\section{Discussion}

In this study, we demonstrate system-wide engineering and optimization of cellular metabolism in C. glutamicum $\mathrm{SO} 1$ to further improve strain performance with respect to L-ornithine biosynthesis. Depending on the current knowledge about L-ornithine biosynthesis pathway, cg3035 was overexpressed using a plasmid that significantly improved L-ornithine production. This confirmed our speculation that glutamate acetylation is a rate-limiting step for L-ornithine accumulation after removing the feedback inhibition of ArgR on the $\operatorname{argCJBDF}$ operon. In the incipient fermentation period, the improvement of L-ornithine production was not obvious probably due to slow glucose utilization rate. In addition, we induced genome-based cg3035 overexpression to ensure strain stability, which also promote L-ornithine accumulation. The improved percentage in ornithine production by genome-based cg3035 overexpression was lower than plasmid based cg3035 overexpression probably due to the not superior NAGS enzyme activity. We believe that this intriguing target could be extended to $\mathrm{L}$-arginine and $\mathrm{L}$-citrulline production.

Notably, $\mathrm{N}$-acetylglutamate biosynthesis also requires acetyl-CoA as a precursor in addition to $\mathrm{N}$-acetylglutamate synthase. Therefore, we attenuated the acetate biosynthesis pathway to reduce acetyl-CoA consumption and improved L-ornithine production titer, thus indicated that acetyl-CoA supplementation is an important factor for L-ornithine production, which further confirmed previous reports that deletion of $d t s R 1$ encoding a subunit of acetyl-CoA carboxylase is beneficial for L-glutamate [25] and L-arginine accumulation [26].This result is also consistent with previous work reported that block the acetate biosynthesis pathway promote the production of several compounds [27-30]. Attenuating acetate biosynthesis also conserves carbon flux into the glycolytic pathway, which may also account for the high L-ornithine production performance. This encouraged us to explore the glycolytic pathways. Overexpression of $p f k A$ by the insertion of a strong $\mathrm{P}_{\text {eftu }}$ promoter tremendously promoted L-ornithine production, whereas insertion of a strong $\mathrm{P}_{\text {eftu }}$ promoter in front of gap or pyk did not contribute to L-ornithine accumulation, which is in contrast with previous results [22]. The differences in genetic background between C. glutamicum ATCC 13032 and C. glutamicum S9114 may account for these diverse outcomes. There is a one percent gap in the genomes of those two strains. In addition, C. glutamicum
S9114, a mutant strain derived from Brevibacterium tianjinese T6-13, processed a faster cell growth than $C$. glutamicum ATCC 13032. Modulations in pyk and gap slightly affected cell growth and glucose consumption in C. glutamicum S9114 probably acting as the main reason for the reduced L-ornithine yield. Based on the aforementioned results, $P_{t a c}-c g 3035, P_{\text {eftu }}-p f k A, T-p t a$, and $T$-cat were confirmed to independently increase L-ornithine accumulation. To further improve the yield of L-ornithine, these targets were assembled. The highest performance was obtained in strain SO12 with $P_{t a c}$ cg3035, $P_{\text {eftu }}-p f k A$, and T-pta, but not in strain SO13 with $P_{t a c}-c g 3035, P_{\text {eftu }}-p f k A, T-p t a$, and T-cat, which illustrated that attenuating $p t a$ and cat simultaneously can affect L-ornithine production by interfering with normal physiological metabolism in engineered strains.

Several previous works have demonstrated that NADPH availability is strongly correlated with L-ornithine production. In this work, L-ornithine production was improved by suppositional redirecting the carbon flux to the pentose phosphate pathway according to previous study [12], which further demonstrated the importance of NADPH supplementation in developing L-ornithine producing strains. This is consistent with previous work by Kim et al., where the pentose phosphate pathway was reinforced by replacing the native promoter of the tkt operon with a strong sod promoter and changing the start codons of $z w f$ and $p g i$ [12].

In summary, compared with previous studies shown in Table 3, this study provide more systematic and comprehensive steps in the construction of an engineered high L-ornithine producing C. glutamicum S9114 strain. Currently, the highest L-ornithine production titer $(51.5 \mathrm{~g} / \mathrm{L})$ was reported by Kim et al. [12], which employ a bioreactor for fed-batch fermentation (Table 3). However, L-ornithine production yield per gram glucose via fed-batch fermentation $(0.24 \mathrm{~g} / \mathrm{g})$ was lower than that of shake flask batch fermentation $(0.524 \mathrm{~g} / \mathrm{g})$ developed by Jensen et al. [10]. In the following work, we will try more strategies such as releasing feedback inhibition of $\arg B$, which is probably the main reason why L-ornithine yield in this study was lower than $0.524 \mathrm{~g} / \mathrm{g}$ glucose, and continue to modulate the metabolic pathway to further improve the production titer of L-ornithine. In addition, inspired by previous work [12], employ a fermentation bioreactor and change the fermentation mode to fedbatch cultivation was supposed to promote L-ornithine accumulation.

\section{Conclusion}

Redesigning and engineering strains for use in the industrial production of L-ornithine has significant potential application for reducing the economic cost pressures 
Table 3 Comparison of other C. glutamicum strains engineered for L-ornithine production

\begin{tabular}{|c|c|c|c|c|c|}
\hline Strains (C. glutamicum) & $\begin{array}{l}\text { L-Ornithine } \\
\text { titer }(g / L)\end{array}$ & $\begin{array}{l}\text { L-Ornithine } \\
\text { yield }(g / g \\
\text { glucose })\end{array}$ & Cultivation & Modulations & References \\
\hline SO16 & 32.3 & 0.395 & Shake flask; batch & $\begin{array}{l}\text { Deletion of } \arg F, n c g / 1221, \arg R, \text { and putP; attenuation } \\
\text { of odhA, proB, pta, cat and } n c g / 2228 ; \text { overexpression } \\
\text { of lysE, gdh, cg3035, pfkA, pyk, tkt, and argCJBD }\end{array}$ & This study \\
\hline YW06 (pSY223) & 51.5 & 0.240 & Bioreactor; fed-batch & $\begin{array}{l}\text { Deletion of } \arg F \text {, } \arg R \text {, and proB; Reinforcement of the } \\
\text { PPP pathway flux; The use of a feedback-resistant } \\
\text { enzyme }\end{array}$ & [12] \\
\hline ORN6 & $20.96^{\mathrm{a}}$ & 0.524 & Shake flask; batch & $\begin{array}{l}\text { Deletion of } \arg F, \arg R \text {, and } \arg G \text {; overexpression of } \\
\arg B^{M} \text {; attenuation of } p g i .\end{array}$ & [10] \\
\hline $\begin{array}{l}\text { SJC8039 } \Delta n c g \mid 0281 \Delta n c g l \\
2582 \Delta n c g / 2053\end{array}$ & $14^{\mathrm{a}}$ & ND & Shake flask; batch & $\begin{array}{l}\text { Deletion of } \operatorname{argF}, \arg R \text {, and proB; Blocking gluconate } \\
\text { biosynthesis }\end{array}$ & [11] \\
\hline$\triangle$ APE6937R42 & 24.1 & 0.298 & Bioreactor; batch & $\begin{array}{l}\text { Deletion of } \arg F \text {, } \arg R \text {, and proB; Adaptive evolution in } \\
\text { presence of } \mathbf{L} \text {-ornithine }\end{array}$ & [13] \\
\hline 1006 $\Delta$ argR-argJ & 31.6 & 0.396 & Shake flask; batch & Deletion of $\arg R$; overexpression of argJ. & {$[31]$} \\
\hline
\end{tabular}

a These values were not described in the main text of the original reference and thus estimated from the figure or graph

of using L-arginine for enzymatic reaction and extending the fermentation organism. C. glutamicum has been extensively studied for its ability to produce L-ornithine, though its yield and productivity are still low compared to other strategies. Very recently, we reported a C. glutamicum S9114 derived recombinant strain with high L-ornithine production titer. Here, we have further enhanced the L-ornithine yield through genome-based cg3035 overexpression and systematic manipulation of central metabolic pathways including glycolysis, acetate metabolism, and pentose phosphate pathway, consequently suggesting that improved L-ornithine production can be obtained by these modulations. C. glutamicum is an excellent producer for producing L-glutamate and L-glutamate-derived products including L-ornithine, $\mathrm{L}$-citrulline, and L-arginine. We supposed that the metabolic engineering strategies reported in this work can be applied to constructing strains producing such products.

\section{Additional file}

Additional file 1: Table S1. Primers and their sequences in this study. Table S2. Promoter and terminator sequence used in this study. Figure

S1. L-Ornithine standard curve of colorimetric assay using ninhydrin.

\section{Authors' contributions}

BZ planned and conducted the experiments, analyzed and interpreted data, and wrote the manuscript; MY and W-PW participated in the research; B-CY supervised the research and finalized the manuscript. All authors read and approved the final manuscript.

\section{Author details}

${ }^{1}$ Laboratory of Biosystems and Microanalysis, State Key Laboratory of Bioreactor Engineering, East China University of Science and Technology, Shanghai 200237, China. ${ }^{2}$ Collaborative Innovation Center of Yangtze River Delta Region Green Pharmaceuticals, College of Pharmaceutical Sciences, Zhejiang University of Technology, Hangzhou 310014, Zhejiang, China.

\section{Acknowledgements}

We thank Dr. Xue-Lan Chen for providing pk18mobsacB. We thank Dr. ZhongGui Mao and Dr. Li-Ming Liu for providing strains Corynebacterium glutamicum S9114. Thank my wife Yan Jiang for giving great support and encouragement during my research.

\section{Competing interests}

The authors declare that they have no competing interests.

\section{Availability of data and materials}

Gene sequences used in this project are from Genbank (http://www.ncbi.nlm. nih.gov/) and the material and data supporting their findings can be found in the main paper and in additional file.

\section{Ethics approval and consent to participate}

All procedures performed in studies involving human participants were in accordance with the ethical standards of the institutional and/or national research committee and with the 1964 Helsinki declaration and its later amendments or comparable ethical standards.

\section{Informed consent}

Informed consent was obtained from all individual participants included in the study.

\section{Funding}

This work was supported by grants from the National Natural Science Foundation of China (31730004 and 21575089).

\section{Publisher's Note}

Springer Nature remains neutral with regard to jurisdictional claims in published maps and institutional affiliations.

Received: 6 March 2018 Accepted: 8 June 2018

Published online: 13 June 2018

\section{References}

1. Becker J, Lange A, Fabarius J, Wittmann C. Top value platform chemicals: bio-based production of organic acids. Curr Opin Biotechnol. 2015:36:168-75.

2. Lee JH, Wendisch VF. Production of amino acids - genetic and metabolic engineering approaches. Bioresour Technol. 2017:245:1575-87. 
3. D'Este M, Alvarado-Morales M, Angelidaki I. Amino acids production focusing on fermentation technologies-a review. Biotechnol Adv. 2017;36:14-25.

4. Nguyen AQ, Schneider J, Reddy GK, Wendisch VF. Fermentative production of the diamine putrescine: system metabolic engineering of Corynebacterium glutamicum. Metabolites. 2015;5:211-31.

5. Meiswinkel TM, Rittmann D, Lindner SN, Wendisch VF. Crude glycerolbased production of amino acids and putrescine by Corynebacterium glutamicum. Bioresour Technol. 2013;145:254-8.

6. Kang M-K, Eom J-H, Kim Y, Um Y, Woo HM. Biosynthesis of pinene from glucose using metabolically-engineered Corynebacterium glutamicum. Biotechnol Lett. 2014;36:2069-77.

7. Siebert D, Wendisch VF. Metabolic pathway engineering for production of 1,2-propanediol and 1-propanol by Corynebacterium glutamicum. Biotechnol Biofuels. 2015;8:91

8. Salvatore F, Cimino F, d'Ayello-Caracciolo M, Cittadini D. Mechanism of the protection by L-ornithine-L-aspartate mixture and by L-arginine in ammonia intoxication. Arch Biochem Biophys. 1964;107:499-503.

9. Shi HP, Fishel RS, Efron DT, Williams JZ, Fishel MH, Barbul A. Effect of supplemental ornithine on wound healing. J Surg Res. 2002;106:299-302.

10. Jensen JV, Eberhardt D, Wendisch VF. Modular pathway engineering of Corynebacterium glutamicum for production of the glutamate-derived compounds ornithine, proline, putrescine, citrulline, and arginine. J Biotechnol. 2015;214:85-94.

11. Hwang $\mathrm{G}-\mathrm{H}$, Cho J-Y. Enhancement of L-ornithine production by disruption of three genes encoding putative oxidoreductases in Corynebacterium glutamicum. J Ind Microbiol Biotechnol. 2014:41:573-8.

12. Kim SY, Lee J, Lee SY. Metabolic engineering of Corynebacterium glutamicum for the production of L-ornithine. Biotechnol Bioeng. 2015;112:416-21.

13. Jiang L-Y, Chen S-G, Zhang Y-Y, Liu J-Z. Metabolic evolution of Corynebacterium glutamicum for increased production of L-ornithine. BMC Biotechnol. 2013;13:47.

14. Zhang B, Ren L, Yu M, Zhou Y, Ye B. Enhanced L-ornithine production by systematic manipulation of $\mathrm{L}$-ornithine metabolism in engineered Corynebacterium glutamicum S9114. Bioresour Technol. 2017;250:60-8.

15. Zhang B, Yu M, Zhou Y, Li Y, Ye B. Systematic pathway engineering of Corynebacterium glutamicum S9114 for L-ornithine production. Microb Cell Fact. 2017;16:158

16. Shäfer A, Tauch A, Jäger W, Kalinowski J, Tierbach G, Pühler A. Small mobilizable multi-purpose cloning vectors derived from the Escherichia coli plasmids pK18 and pK19: selection of defined deletions in the chromosome of Corynebacterium glutamicum. Gene. 1994;145:69-73.

17. Zhang B, Wan F, Qiu Y, Chen X, Tang L, Chen J, Xiong Y. Increased L-arginine production by site-directed mutagenesis of $N$-acetyl-L-glutamate kinase and proB gene deletion in Corynebacterium crenatum. Biomed Environ Sci. 2015;28:864-74.

18. Zhang B, Yu M, Zhou Y, Ye BC. Improvement of L-ornithine production by attenuation of arg $\mathrm{F}$ in engineered Corynebacterium glutamicum $\mathrm{S} 9114$. AMB Express. 2018;8:26.

19. Petri K, Walter F, Persicke M, Ruckert C, Kalinowski J. A novel type of $\mathrm{N}$-acetylglutamate synthase is involved in the first step of arginine biosynthesis in Corynebacterium glutamicum. BMC Genom. 2013;14:713.

20. Liao CH, Yao L, Xu Y, Liu WB, Zhou Y, Ye BC. Nitrogen regulator GInR controls uptake and utilization of non-phosphotransferase-system carbon sources in actinomycetes. Proc Natl Acad Sci USA. 2015;112:15630-5.

21. Rosen $\mathrm{H}$. A modified ninhydrin colorimetric analysis for amino acids. Arch Biochem Biophys. 1957;67:10-5.

22. Jiang L-Y, Zhang Y-Y, Li Z, Liu J-Z. Metabolic engineering of Corynebacterium glutamicum for increasing the production of L-ornithine by increasing NADPH availability. J Ind Microbiol Biotechnol. 2013:40:1143-51.

23. Qiu Z, Gao Q, Bao J. Engineering Pediococcus acidilactici with xylose assimilation pathway for high titer cellulosic L-lactic acid fermentation. Bioresour Technol. 2018;249:9-15.

24. Rytter JV, Helmark S, Chen J, Lezyk MJ, Solem C, Jensen PR. Synthetic promoter libraries for Corynebacterium glutamicum. Appl Microbiol Biotechnol. 2014;98:2617-23.

25. Yao W, Deng X, Zhong H, Liu M, Zheng P, Sun Z, Zhang Y. Double deletion of dtsR1 and pyc induce efficient L-glutamate overproduction in Corynebacterium glutamicum. J Ind Microbiol Biotechnol. 2009;36:911-21.

26. Chen M, Chen X, Wan F, Zhang B, Chen J, Xiong Y. Effect of tween 40 and DtsR1 on L-arginine overproduction in Corynebacterium crenatum. Microb Cell Fact. 2015;14:119.

27. Feng $L$, Zhang $Y, F u$ J, Mao Y, Chen T, Zhao X, Wang Z. Metabolic engineering of Corynebacterium glutamicum for efficient production of 5-aminolevulinic acid. Biotechnol Bioeng. 2016:113:1284-93.

28. Balagurunathan B, Tan L, Zhao H. Metabolic engineering of Escherichia coli for acetaldehyde overproduction using pyruvate decarboxylase from Zymomonas mobilis. Enzyme Microb Technol. 2018;109:58-65.

29. Kim SK, Seong W, Han GH, Lee DH, Lee SG. CRISPR interference-guided multiplex repression of endogenous competing pathway genes for redirecting metabolic flux in Escherichia coli. Microb Cell Fact. 2017;16:188.

30. Harder BJ, Bettenbrock K, Klamt S. Model-based metabolic engineering enables high yield itaconic acid production by Escherichia coli. Metab Eng. 2016;38:29-37.

31. Hao N, Mu J, Hu N, Xu S, Shen P, Yan M, Li Y, Xu L. Implication of ornithine acetyltransferase activity on L-ornithine production in Corynebacterium glutamicum. Biotechnol Appl Biochem. 2016;63:15-21.
Ready to submit your research? Choose BMC and benefit from:

- fast, convenient online submission

- thorough peer review by experienced researchers in your field

- rapid publication on acceptance

- support for research data, including large and complex data types

- gold Open Access which fosters wider collaboration and increased citations

- maximum visibility for your research: over 100M website views per year

At BMC, research is always in progress.

Learn more biomedcentral.com/submissions 